\title{
Analysis of Pedestrian-Fatality Statistics in Japan and the US and Vehicle-Pedestrian Communication for Vehicle-Pedestrian Crash-Warnings
}

\author{
Hirofumi Onishi ${ }^{\text {1) }}$ Takeshi Hirai $^{2)}$ Ryoya Kawasaki $^{2)}$ Hayami Ito ${ }^{\text {3) }}$ Tutomu Murase ${ }^{2)}$ \\ 1) Alpine Electronics Research of America, Inc. 19145 Gramercy Pl., Torrance, CA 90501 USA (honishi@alpine-la.com) \\ 2) Nagoya University Furo-cho, Chikusa-ku, Nagoya, 464-8601 Japan \\ 3) Tokyo Institute of Technology 2-12-1 Ookayama, Meguro-ku Tokyo 152-8550 Japan
}

Received on July 5, 2017

\begin{abstract}
Vehicle-pedestrian crashes are more likely to result in fatalities or severe injuries as compared to vehicle-vehicle crashes. Therefore, various vehicle-pedestrian crash-warning solutions have been proposed. In this report, we first analyze pedestrian-fatality-statistics in the world, Japan, and the US; we then examine various vehicle-pedestrian crash-warning solutions, including three types of communication-solutions that can detect pedestrians in non-line-of-sight: "pedestrians' communication-modules communicate to vehicles directly", "vehicles' modules detect pedestrians' mobile-phones", and "vehicles and pedestrians communicate through mobile-network". Next, we analyze technical challenges in communicationsolutions: channel-congestion, communication-delay, and others. Finally, we conclude this report with approaches to implement communication-solutions for vehicle-pedestrian crash-warnings.
\end{abstract}

KEY WORDS: information, communication, and control, Communication, Vehicle-to-vehicle communication, Accident avoidance/collision prediction, Pedestrian detection, Pedestrian traffic fatalities,

Vehicle-to-pedestrian communication [E2]

\section{Pedestrian Traffic Fatality Statistics}

According to the World Health Organization, in 2010, 273,000 pedestrians ( $22 \%$ of all road traffic fatalities) were killed in the world, and almost half of traffic fatalities are vulnerable road users, such as pedestrians, cyclists, and motorcyclists (Figure 1) (1) $^{(1)}$ In Japan, thanks to advanced vehicles safety technologies, in 2015 the decrease of vehicle drivers/occupants fatalities created a relative increase in the ratio of pedestrian fatalities $(37 \%$ of all road traffic fatalities) (Fig. 1 and Fig. 2) ${ }^{(2)}$, compared to pedestrian fatalities in 2005 (31\%). As the left diagram (a) of Fig. 3 shows, pedestrian fatalities at crossings $(26 \%)$ and pedestrian fatalities by other vehicle-pedestrian crashes (11\%) comprise a large segment of total traffic fatalities in Japan.

The right diagram (b) of Fig. 3 shows pedestrian fatalities at crossing roads occurring mainly at intersections (51\%) and near intersections (17\%).

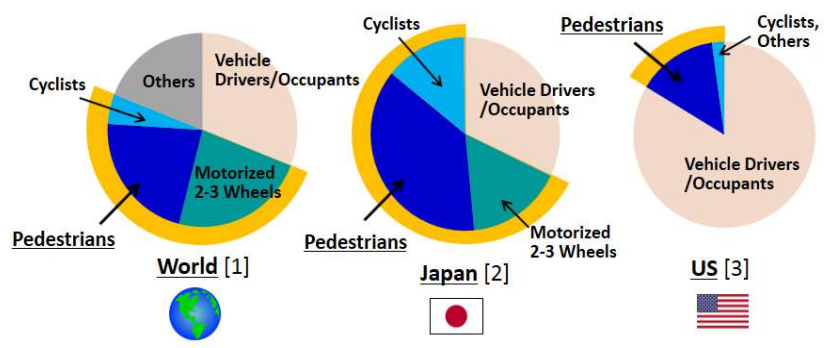

Fig. 1 Traffic Fatality by Mode in the World, Japan, and the US

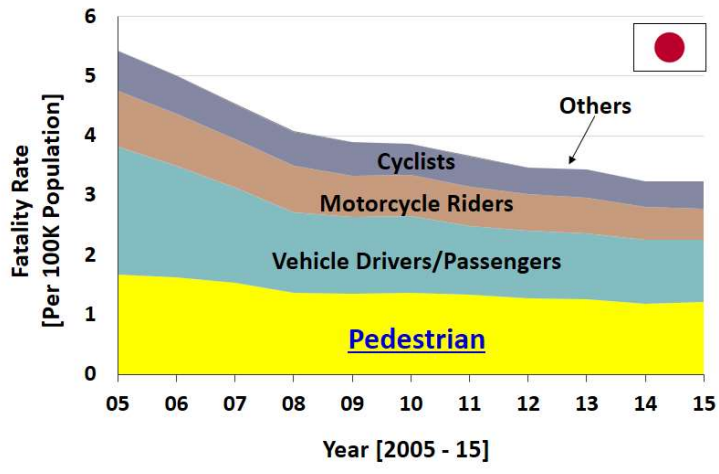

Fig. 2 Traffic Fatality by Mode in Japan (from 2005 to 2015)
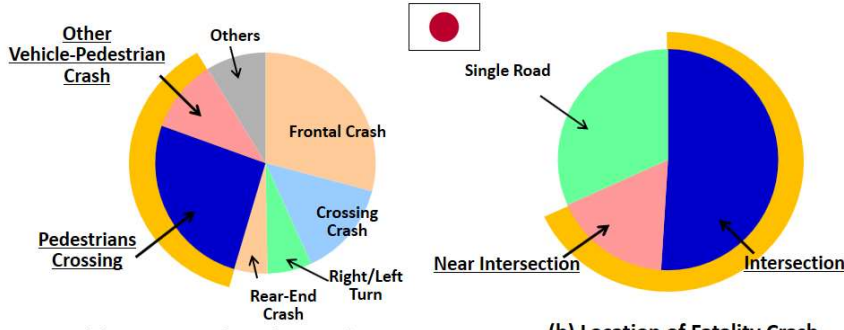

(a) Situation of Fatality Crash

Fig. 3 Detailed Breakdown in Traffic Fatality in Japan ${ }^{(2)}$

(C)2018 Society of Automotive Engineers of Japan, Inc. This is an open access article under the terms of the Creative Commons Attribution-NonCommercial-ShareAlike license. 
Even in the most motorised country in the world, the U.S., 4,700 pedestrians (14\% of the total traffic fatalities) were killed in 2013 (Figure 1$)^{(3)}$, and $76 \%$ of pedestrian fatalities were the result of being hit by a vehicle in dark conditions ${ }^{(3)} .73 \%$ of total pedestrian fatalities were killed in urban areas. In fact, pedestrian fatalities occupy nearly half of total traffic fatalities in large cities, for example, the city of New York (61\%), San Francisco (55\%), Washington DC (45\%), Boston (44\%), San Jose (40\%), Detroit $(37 \%)$, and others ${ }^{(3)}$. Note the large difference in pedestrian fatality statistics between Japan and the US: in the US, the ratio of pedestrian fatalities at intersections of total pedestrian fatalities is only $20 \%^{(3)}$ (Fig. 4 ), though it comprises $51 \%$ in Japan.

Fig. 5 shows a significant finding regarding pedestrian fatalities in the US: Though $40 \%$ of total traffic fatalities are related to drunk driving (the right picture of Fig. 5), only $20 \%$ of pedestrian fatality crashes are related to drunk-driving. This result indicates: almost of crashes with pedestrian fatalities were caused, in spite of drivers' cautious driving.

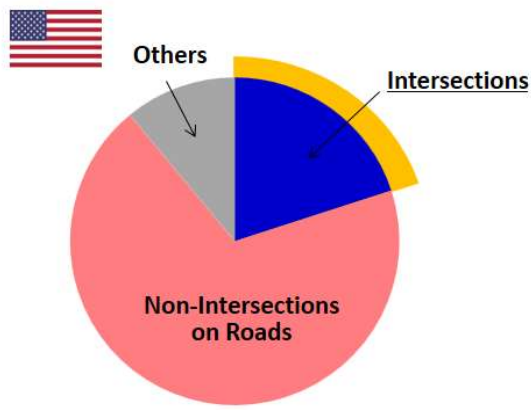

Fig. 4 Locations of Pedestrian Fatalities in the US ${ }^{(3)}$
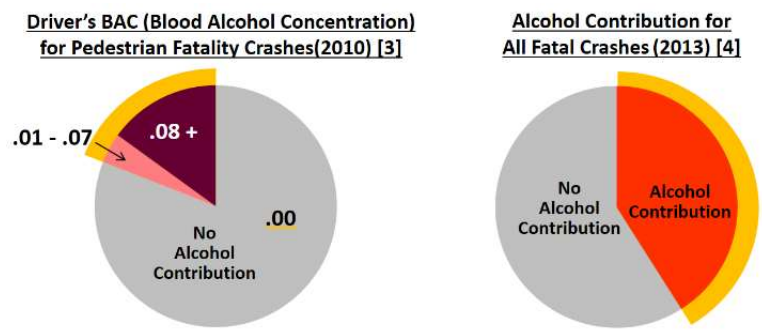

Fig. 5 Pedestrian-Fatalities and Drunk Driving in the US ${ }^{(3)(4)}$

\section{Public Initiatives to Protect Vulnerable Road Users}

To reduce vulnerable road user fatalities (including pedestrians), governments and public organizations are taking various actions, for example:

- The United Nations launched an initiative titled "Decade of Action for Road Safety 2011-2020"(5).

- The Ministry of Internal Affairs and Communications in Japan solicited ICT solutions to avoid crashes between pedestrians/cyclists and vehicles, in July $2014^{(6)}$.

- The U.S. Department of Transportation declared a "Bike/Pedestrian Safety Initiative" in 2014 ${ }^{(7)}$

- The European Commission completed a research project, "VRUITS (Vulnerable Road Users - ITS)" in March 2016 ${ }^{(8)}$.
Additionally, vehicle product guidelines started to focus on pedestrian protection in Europe and the US, such as,

- The U.S. Department of Transportation published AEB (Automatic Emergency Braking) guidelines geared mainly at the safety of pedestrians or cyclists ${ }^{(9)}$.

- Euro-NCAP included pedestrian safety in its vehicle safety ranking ${ }^{(10)}$.

\section{Solutions for Vehicle-Pedestrian Crash Warnings ${ }^{(11)}$}

Because vehicle pedestrian crashes more frequently result in pedestrian fatalities or severe pedestrian injuries compared to crashes only involving vehicles, the automotive and ITS industries have researched various solutions for vehicle-pedestrian crash warning. Infrastructure assist systems can provide high performance without specific devices for pedestrians or vehicles even in limited lighting conditions ${ }^{(12)(13)}$. Devices on vehicles can typically make drivers aware of dangers more effectively. However, this type of solutions works only where infrastructure systems are installed (Table 1). In other types of solutions, communication solutions for vehicle pedestrian crash warning have the following significant benefits compared to sensor solutions (for example, visual/infrared cameras or radars) (Table 1):

- to detect pedestrians in non-line-of-sight, i.e. drivers' blind spots.

- to operate stably in spite of ambient lighting or weather conditions.

In communication solutions, there are three typical approaches are:

A. have pedestrians carry V2X (vehicle-to-everything, such as vehicle-to-vehicle, vehicle-to-road, etc) communication devices to communicate to vehicles directly ${ }^{(14)(15)}$.

B. install radio detectors in vehicles to detect locations of pedestrians' mobile-phones.

C. enable neighboring vehicles and pedestrians to communicate through mobile-communication ${ }^{(16)}$.

Table 1 shows the comparison of individual solutions. Mobilephone detection (item B above) requires complicated antennastructures, in order to detect locations of pedestrians' mobilephones.

Table 1 Solutions of Vehicle-Pedestrian Crash Warnings ${ }^{(11)}$

\begin{tabular}{|c|c|c|c|c|}
\hline \multirow{2}{*}{\multicolumn{2}{|c|}{ Approach }} & \multirow{2}{*}{ Regions } & \multicolumn{2}{|c|}{ Equipment } \\
\hline & & & Vehicle & Pedestrian \\
\hline \multicolumn{2}{|r|}{$\begin{array}{l}\text { Infrastructure } \\
\text { Assist }\end{array}$} & Limited & $\begin{array}{l}\text { Not } \\
\text { Necessary }\end{array}$ & Not Required \\
\hline \multicolumn{2}{|r|}{$\begin{array}{c}\text { Sensor: } \\
\text { Camera, Radar, etc }\end{array}$} & Not-limited & Required & Not Required \\
\hline \multirow{3}{*}{ 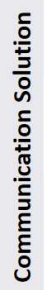 } & V2X: DSRC & Limited & Ordinary V $2 \mathrm{X}$ & $\begin{array}{l}\text { Required: } \\
\text { Not difficult }\end{array}$ \\
\hline & $\begin{array}{l}\text { Mobile-phone } \\
\text { Detection }\end{array}$ & $\begin{array}{l}\text { Required: } \\
\text { Mobile-phones }\end{array}$ & $\begin{array}{l}\text { Special } \\
\text { detector }\end{array}$ & $\begin{array}{l}\text { Ordinary } \\
\text { mobile- } \\
\text { phone }\end{array}$ \\
\hline & $\begin{array}{l}\text { Mobile- } \\
\text { communication } \\
\text { (through Server) }\end{array}$ & $\begin{array}{l}\text { Required: } \\
\text { Mobile- } \\
\text { communication }\end{array}$ & \multicolumn{2}{|c|}{$\begin{array}{l}\text { Ordinary mobile-phone } \\
\text { with data service }\end{array}$} \\
\hline
\end{tabular}


Mobile-phone communication (item $\mathrm{C}$ above) has mainly two solutions. One solution is: information (location/speed/direction) of vehicles/pedestrians are transmitted to the specific server, then the server transmits the messages to vehicles/pedestrians that have crash risks because of neighboring vehicles/pedestrians ${ }^{(16)}$. However, this solution still has difficulties to be deployed to timesensitive crash warning applications, even with LTE (Long Term Evolution), because of communications latency (typically over 100 milliseconds as end-to-end) ${ }^{(17)}$.

The other solution is: all vehicles/pedestrians transmit their information (location/speed/direction) to a base-station of mobilenetwork, then the base station transmits all received information to all vehicles/pedestrians in the cell (i.e. areas covered by this basestation) ${ }^{(18)}$. However, we need assist from base-stations of mobilenetworks in order to implement this solution, furthermore, we have to evaluate whether a base-station has sufficient performance to cover all vehicles/pedestrians in the cell with considering the available bandwidth of wireless spectrum. In this base-station assisted solution, vehicles/pedestrians in the cell edge require the information from vehicles/pedestrians in neighbour cells, in order to detect potential crashes, therefore, we need more complicated algorithm in practice.

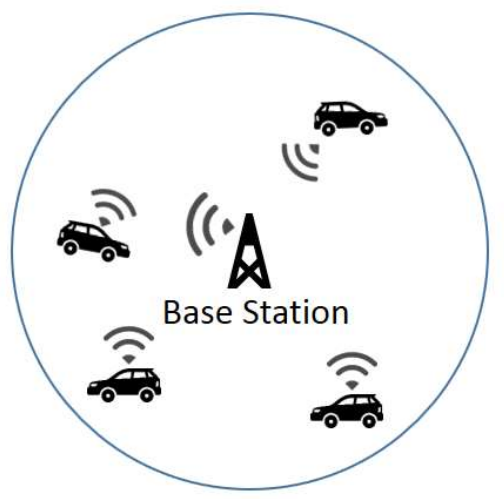

Fig. 6 Concept of V2X with LTE by Base-Station Assist ${ }^{(18)}$

As the result, in regions where V2X is deployed for vehicles, the $\mathrm{V} 2 \mathrm{X}$ extension for V2P (vehicle-to-pedestrian) (item A above) is the most feasible way to implement vehicle-pedestrian crash warnings. (However, this approach is not feasible in regions where vehicles do not have V2X devices.)

\section{Challenges for Vehicle-to-Pedestrian Communication}

Despite the promise of V2X communication solutions, it still poses the following technical challenges for application to vehiclepedestrian crash warnings:

a. radio propagation ${ }^{(19)}$

b. communications latency

c. channel congestion ( scalability)

d. energy consumption of pedestrian devices (if additional consumption is required) $)^{(14)}$

\subsection{Radio propagation}

In spite of a large benefit of communications solutions, i.e., to detect pedestrians in non-line-of-sight (in other words, drivers' blind spots), blockages on a communication path (such as, trucks, buses, large buildings, or trees) may disturb communications between pedestrians and vehicles. However, fortunately, the large output power $(20 \mathrm{dBm})$ of V2X (as defined by the U.S. Department of Transportation ${ }^{(20)}$ ), compared to ordinary WiFi output power of laptops $(18 \mathrm{dBm})$, can overcome ordinary blockages (trucks and busses) on roads. In two examples of our previous experiments for communications beyond blockages (Fig. 7), we did not observe any communication failures ${ }^{(21)}$.
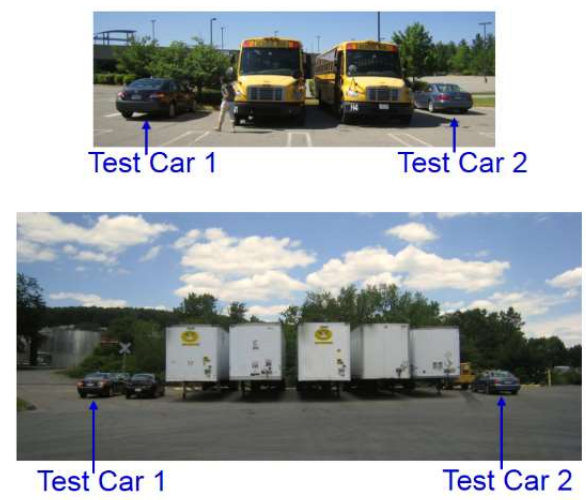

Measured by octoScope/Alpine on Jun. 2013 (in Marlboro, MA)

Fig. 7 V2X Radio Propagation Experiments ${ }^{(21)}$

4.2. Communication latency and channel congestion

V2X defined by IEEE $802.11 \mathrm{p}$ has sufficiently short communication latency $(0.2-15$ milliseconds $)$ for crash warning (compared to drivers' physical reactions against crash risks). However, the IEEE 802.11p protocol with CSMA (Carrier Sense Multiple Access) ${ }^{(22)}$ needs a longer time to transmit a message within areas crowded with a large number of communication nodes, because of retransmission or back-off caused by channelcongestion. In our previous analysis, V2X communication protocols allow approximately 150 neighboring vehicles to exchange their location/speed/direction every 100 milliseconds for vehicle-vehicle crash warning applications ${ }^{(23)(24)}$ (Fig. 8). Though this is a reasonable number for vehicles within a typical V2X communication range (approximately 300 meters), we need to accommodate more nodes to deploy vehicle-to-pedestrian communication in areas crowded with vehicles and pedestrians, such as city centers.

Furthermore, regardless of traffic regulations, pedestrians have freedom of movements, such as large speed changes and large direction changes for a short duration, in spite of their slower speeds (compared to vehicles). Therefore, it may not be appropriate to reduce data transmission frequency from the current data transmission frequency $(10 \mathrm{~Hz})$ defined for vehicle-to-vehicle crash warnings.

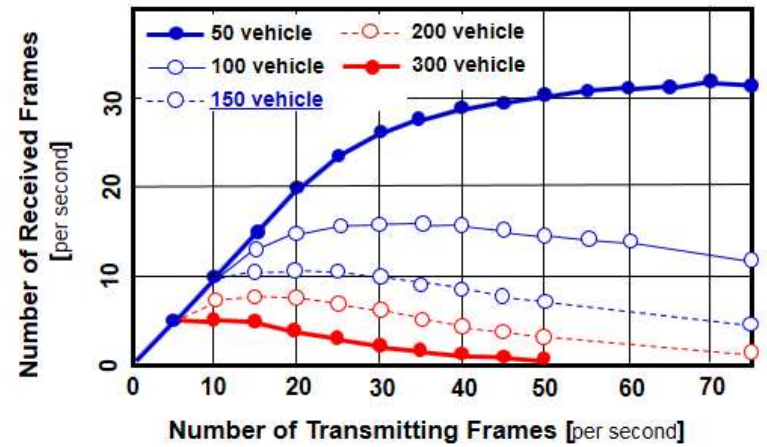

Fig. 8 V2X Channel Congestion Evaluation ${ }^{(24)}$ 


\subsection{Energy consumption of pedestrian devices}

Finally, while V2X's large output power $(20 \mathrm{dBm})$ and the data transmission frequency (potentially $10 \mathrm{~Hz}$ ) mitigate the issues of "radio propagation (item a above)" and "communications latency (item b above)", these approaches also affect "the energy consumption of pedestrian devices (item $d$ above)". Per a previous study ${ }^{(14)}$, V2X messages defined for vehicle-to-vehicle crash warnings consume the energy of ordinary smartphones' batteries within only a few hours.

\section{Approaches to Implement V2P Communication}

\subsection{Control data transmission frequency}

As Fig. 9 (a) shows, generally, slower speeds of pedestrians (compared to vehicles) allow lower data transmission frequency for vehicle-pedestrian crash warnings than for vehicle-vehicle crash warnings. This lower data transmission frequency mitigates "channel congestion (item c above)" and leads to the reduction of "communications latency (item b above)". Furthermore, if we reduce data transmission frequency, we can accommodate more nodes (i.e. total number of vehicles and pedestrians). Additionally, this lower data transmission frequency decreases "energy consumption of pedestrians' devices (item d above)". The reduction of data transmission frequency from $10 \mathrm{~Hz}$ to $5 \mathrm{~Hz}$ almost doubles battery power endurance.

Responding to pedestrians' sudden direction/speed changes, we can allow higher data transmission frequency only at the moments of pedestrians' sudden direction/speed changes (situations with () red-circles in Fig. 9 (b)).

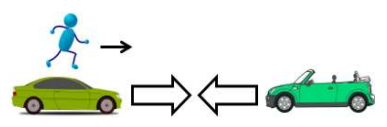

(a)

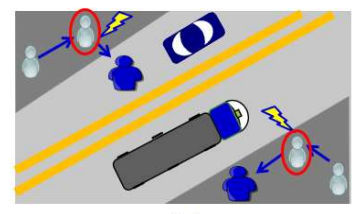

(b) (those receiving pedestrian (1)'s message) within the predefined distance (for example, 5 meters) between the pedestrian (1) may stop transmitting messages for the predefined duration (for example, 0.1 seconds). This approach can mitigate "channel congestion (item c above)" and "communications latency (item b above)" by reducing individual pedestrian devices' data-frame transmissions, and this approach can also reduce "battery power consumption of pedestrian devices (item d above)".

Fig. 10 (b) shows our preliminary calculations for the condition of a head-on crash between a vehicle at $40 \mathrm{~km} / \mathrm{h}$ and a stopped pedestrian, while all vehicles and pedestrians in the communication-range $(300 \mathrm{~m})$ transmit data-frames defined by V2V for vehicle-vehicle crash warning at $20 \mathrm{~Hz}$. The result shows: grouping approach increased $30 \%$ of additional nodes that could exchange data-frames, 2.5 seconds prior to potential crashes. We calculated this result under uniform vehicle/pedestrian density though pedestrians are subject to gather at near intersections or pedestrian crossings in real roads. Therefore, this grouping approach has a possibility to accommodate over $30 \%$ additional nodes (such as the total number of vehicles and pedestrians) in the real world ${ }^{(25)}$. In our calculation, on average 1.2 nodes compose one group, such that the number of transmitted data-frames was reduced by $17 \%$ on average. Since data-frame transmission is a major factor of energy consumption in batteries of mobile-devices, the reduction of transmitted data-frames brings a significant saving of battery energy.

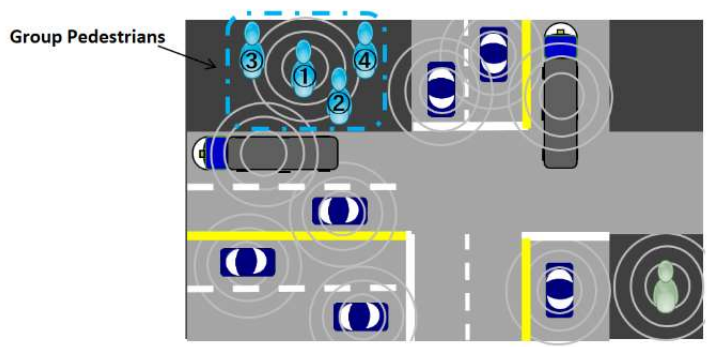

(a) Concept of grouping

Fig. 9 Data Transmission Frequency Control for Vehicle-Pedestrian Communication

\subsection{Group neighboring pedestrians}

Pedestrians close to intersections are more likely to have traffic accidents, especially in Japan ${ }^{(2)}$. Additionally, large numbers of pedestrians are gathering at intersections or pedestrian crossings in urban areas where over $70 \%$ of pedestrians are killed in the US (refer to the chapter 1 in this manuscript $)^{(3)}$. However, since the large number of vehicles and pedestrians gathering in a neighbourhood causes "channel congestion (item c above)" and degrades "communications latency (item b above)", we propose to handle multiple pedestrians gathering in a neighbourhood as one node, instead of handling individual pedestrians as individual nodes.

In the upper-left corner of Fig. 10 (a), the group of four pedestrians can transmit only one message (representing four pedestrians) to mitigate "channel congestion (item c above)" and to reduce "communications latency (item b above)". Furthermore, in many cases, the approach of warning drivers about a group of pedestrians can provide an effective and simple warning to drivers, rather than a warning of many individual pedestrians

As one implementation approach, in Fig. 10 (a), once a pedestrian (1) transmitted a message, pedestrians (2), (3), and (4)

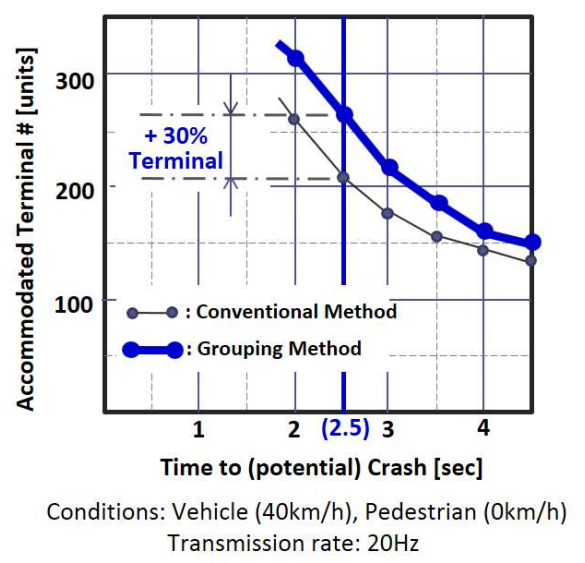

(b) (primary) effect of grouping

Fig. 10 Grouping Neighboring Pedestrians ${ }^{(25)}$ 
Hirofumi Onishi et al./International Journal of Automotive Engineering Vol.9, No.4 (2018) pp.231-236

\section{Conclusion}

Increasing pedestrian fatalities ( $22 \%$ of total traffic fatalities) in the world is a serious social concern. Especially in Japan, pedestrian-fatalities have the largest ratio of all traffic fatalities (37\%).

Therefore, governments, public organizations, and automotive and ITS industries are taking various actions. In particular, industries are proposing various vehicle-pedestrian crash warning solutions. In all types of crash warning solutions, communication solutions have large advantages: to detect a pedestrian in non-lineof-sight and to work effectively regardless of ambient lighting conditions. Especially in many types of communication solutions, to apply V2X communication for vehicle-pedestrian crash warnings is the most feasible solution in the areas where V2X is deployed.

However, even for the most feasible extension of V2X communication, increased communication nodes (i.e. total number of vehicles and pedestrians) in city centers brings channel congestion and communication latency. Furthermore, the limited size of pedestrian devices raises the issue of energy consumption of batteries in pedestrian devices.

To overcome these challenges, we propose the two following approaches:

- Control of data transmission frequency:

to reduce data transmission frequency by utilizing pedestrians' slow speeds (compared to vehicles).

- Node-grouping approach:

to group multiple neighboring pedestrians as one single communication node.

In our proposed grouping approach where nodes (i.e. pedestrians) pause data-frame transmission during a pre-defined duration (e.g. 0.1 seconds) after they receive neighboring node's data-frames, our modeling preliminarily showed we could accommodate an additional $30 \%$ vehicles and pedestrians (compared to 150 nodes by the conventional method).

\section{Additional Challenges: To Detect Pedestrian Locations}

Finally, we address one critical factor for the success of vehiclepedestrian crash warnings by using V2X. Generally, pedestrians can freely locate on roads (for vehicles), pedestrian crosswalks, parking lots, and facilities. Furthermore, pedestrians can more quickly change speeds and directions than vehicles. Therefore, it is a critical challenge to detect pedestrian locations with high accuracy, higher time resolution, and shorter time latency with limited sensors and calculation performance on pedestrian devices.

This paper is written based on a proceeding presented at JSAE 2017 Annual Congress.

\section{Acknowledgement}

The authors express gratitude to Mr. Richard Bishop of Bishop Consulting for his advice and review of our research, especially for this report.

\section{References}

(1) World Health Organization, Pedestrian safety, http://apps.who.int/iris/bitstream/10665/79753/1/9789241505 352_eng.pdf?ua $=1$ (accessed in Mar. 2017)

(2) Japan National Police Agency, Characteristics of traffic fatalities in 2015 (Japanese), http://www.npa.go.jp/toukei/koutuu48/H27_setsumeishiryo.p df (accessed in Mar. 2017)

(3) NHTSA in the U.S. DoT, Traffic Safety Facts 2013 Data, www-nrd.nhtsa.dot.gov/Pubs/812124.pdf (accessed in Mar. 2017)

(4) Bureau of Transportation Statistics U.S. DoT, National Transportation Statistics 2015, www.rita.dot.gov/bts/sites/rita.dot.gov.bts/files/NTS_Entire_1 5Q2_rev.pdf (accessed in Mar. 2017)

(5) United Nations, Decade of Action for Road Safety 2011-2020, www.who.int/roadsafety/projects/en/ (accessed in Mar. 2017)

(6) Ministry of Internal Affairs and Communications Japan, Solicitation of 2014 ICT research proposal, www.soumu.go.jp/menu_news/snews/01tsushin03_02000093.html and www.soumu.go.jp/menu_news/snews/01 tsushin03_02000107.html (accessed in Mar. 2017)

(7) Irvin Dawid, Transportation Secretary Foxx Announces Bike/Ped Safety Initiative, www.planetizen.com/node/71230 (accessed in Mar. 2017)

(8) VRUITS (Vulnerable Road Users - Intelligent Transport Systems), www.vruits.eu/ (accessed in Mar. 2017)

(9) NHTSA, Automatic Emergency Braking in Safercar.gov, www.safercar.gov/Vehicle-Shoppers/SafetyTechnology/AEB/aeb (accessed in Mar. 2017)

(10) Euro-Ncap, Pedestrian Protection, www.euroncap.com/en/vehicle-safety/the-ratingsexplained/pedestrian-protection/ (accessed in Mar. 2017)

(11) U.S. DoT, Vehicle to Pedestrian Technical Scan Summary, www.its.dot.gov/press/2015/v2p tech.htm (accessed in Mar. 2017)

(12) T. Ruß, V2X-based cooperative protection system for vulnerable road users and its impact on traffic, Proceedings of ITS World Congress 2016, Paper\# EU-TP0686 (Melbourne, Australia) (2016)

(13) UTMS Society of Japan, DSSS Driving Safety Support Systems, www.its-jp.org/english/files/2013/10/Nextgeneration-DSSS-leaflet.pdf (accessed in Mar. 2017)

(14) X. Wu, R. Miucic, et al, Cars Talk to Phones: A DSRC Based Vehicle-Pedestrian Safety System, (DOI: 10.1109/VTCFall.2014.6965898) in IEEE VTS (Vancouver, Canada) (2014)

(15) M. Jutila, J. Scholliers, M. Valta, K. Kujanpää, Assessment of the Performance for Vulnerable Road User Safety Applications, Proceedings of ITS World Congress 2015, Paper\# EU-ITS-2464 (Bordeaux, France) (2015)

(16) Nissan, GPS Mobile ITS for Pedestrian Safety, www.nissanglobal.com/EN/TECHNOLOGY/OVERVIEW/gps_mobile_it s.html (accessed in Mar. 2017)

(17) H. Onishi, F. Mlinarsky, Wireless Technology Assessment for Automotive Applications, Proceedings of ITS World Congress 2012, Paper\# AM-00013 (Vienna, Austria) (2012)

(18) R. Kawasaki, T. Hirai, et. al, Performance Evaluation on V2X Communications by LTE for Crash Warning Application, pp.175-180, No.485, IEICE-116, IEICE-IN2016126 (Japanese)(2017)

(19) S. Lee, J. Yoon, S. Min, Study on architecture and application for vehicle to pedestrian communication, Proceedings of ITS World Congress 2015, Paper\# AP-ITS1997 (Bordeaux, France) (2015) 
(20) Dept. of Transportation, (NHTSA-2014-0022-0002) FMVSS: Vehicle-to-Vehicle (V2V) Communications (2014)

(21) H. Onishi, F. Mlinarsky, et al, Wireless Technology Assessment with Radio Channel Emulator, Proceedings of ITS World Congress 2013, Paper\# 1001 (Tokyo, Japan) (2013)

(22) Wikipedia, Carrier sense multiple access, http://en.wikipedia.org/wiki/Carrier_sense_multiple_access (accessed in Mar. 2017)

(23) US Depart of Transportation, (FHWA-JPO-15-218) Status of the DSRC Technology and Applications (2015)

(24) H. Ito, K. Wako, et al, Crash warning for intersection and head-on car collision in vehicle-to-vehicle communication, (DOI: 10.1109/ICCVE.2015.27) in IEEE ICCVE (Shenzhen, China) (2015)

(25) H. Ito, H. Onishi, et al, V2P Communications for V2P CrashWarning, Proceedings of ITS World Congress 2016, Paper\# AM-TP0116 (Melbourne, Australia) (2016) 